\title{
NANOPRINTING OF MINIATURE COMPOUND REFRACTIVE LENSES FOR DESKTOP HARD X-RAY IMAGING
}

\author{
Mona Mirzaeimoghri ${ }^{1,2}$, Alejandro Morales ${ }^{1}$, Caroline McCue ${ }^{2}$, Don L. DeVoe ${ }^{2}$, Han Wen ${ }^{1}$ \\ ${ }^{1}$ Imaging Physics Laboratory, Biochemistry and Biophysics Center, \\ National Heart, Lung and Blood Institute, National Institute of Health, Bethesda, MD \\ ${ }^{2}$ Dept. of Mechanical Engineering, University of Maryland, College Park, MD
}

\begin{abstract}
Focusing hard x-rays for microscopy has been a long standing challenge. Compound refractive lens (CRL) technology represents one approach to addressing this challenge using series of lenses than can accumulate the small refraction of each lens. A novel fabrication approach using a two-photon laser photopolymerization system to pattern epoxy-based CRLs by direct 3D nanoprinting is utilized here to report the first instance of a nano-printed lens for hard x-ray microscopy, with evaluation of its imaging performance. The resulting lens technology was found to enable benchtop microscopy to image and assess the size and stability of the focal spots of tungsten-target micro-focus x-ray sources.
\end{abstract}

\section{INTRODUCTION}

Miniature X-ray optics can create new opportunities for compact imaging systems for use in science, medicine, and technology. Compared to visible light, x-rays have shorter wavelengths which give it the ability to penetrate deeper into matter, making them highly useful for applications in microanalysis and imaging where it is desirable to probe beyond the surface of the material under analysis. Focusing hard x-rays for microscopy has been a challenging task for decades, particularly for small-scale imaging systems. Focusing elements for $\mathrm{x}$-ray microscopy for soft x-ray ( $<10 \mathrm{keV}$ photon energy) include curved mirrors (Kirkpatrick-Baez) in benchtop microscopy [1], zone plates at $8 \mathrm{keV}$ [2] and compound refractive lenses (CRL) which consist of linear arrays of individual refractive lenses [3]. However, complexity of fabrication and limitation of hard x-ray performance make it difficult to achieve an ideal form of focusing optics element.

Compound refractive lens (CRL) technology represents one approach to addressing this challenge that offers good efficiency and robustness by employing a series of focusing elements than can accumulate small refraction changes provided by each lens. Different kinds of linear CRL fabrication processes have been investigated, such as drilling an array of holes in an aluminum block [3] and using electron lithography or deep x-ray lithography to produce CRLs in silicon and polymer. However, two cylindrically-focused CRLs arranged perpendicular to each other are required to form a proper lens for microscopy, leading to directional aberration and doubling of length. A more elegant solution is a truly two-dimensional CRL. The idea has been demonstrated in the form micro-bubbles in epoxy [4], patterned planar lens structures [5],[6], and polymer based CRLs[7]. However, current limitations in the fabrication of $3 \mathrm{D}$ structures limit their use for soft $9 \mathrm{keV}$ x-rays and long focal lengths of $>10$ $\mathrm{cm}$.

\section{Methods and Material Design}

In order to focus a beam to a spot, the exit wavefront should have a spherical profile (Figure 1). To obtain that profile, the beam passing through the polymer and air should have accumulated different phases. Due to fact that the refractive index of the x-ray is less than one, the wavefront is phase shifted forward inside the

polymer. The profile of the lens should produce a spherically shaped wavefront. Referring to Fig. 1 the forward shift of the wave front at spherical radius $R$, for $R<<$ focal length $f$, should be

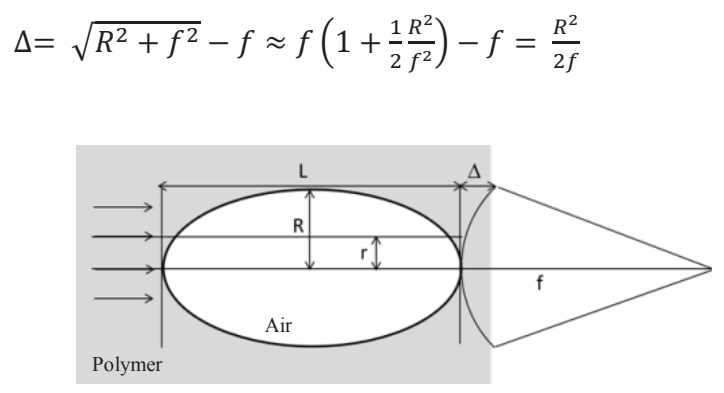

Figure 1. Refraction of a beam passing through a parabolic surface

The refractive index of the lens is $\mathrm{n}=1-\delta$, hence the maximum length $(L)$ necessary for $\Delta$ is given by:

$$
\delta L=\frac{R^{2}}{2 f}
$$

Similarly, for the rest of the structure, the beam equation can be written as:

$$
L(r)=\frac{r^{2}}{2 f \delta}
$$

Which is a parabolic equation. For that reason, in order to produce the spherical wave, a parabolic lens is needed. Therefore, For a CRL made of a series of $N$ concave lenses each having a parabolic surface of length $l$, the focal length is:

$$
f=\frac{R^{2}}{2 N l \delta}
$$

\section{Fabrication}

We present a fabrication process using a two-photon laser photopolymerization system to pattern epoxy-based CRLs by direct 3D nanoprinting. To fabricate the lenses, a droplet of photoresist (IP-DIP) was drop-casted on the edge of the $700 \mu \mathrm{m}$ thick fused silica substrate. Micrometer-scale lenses were patterned in the resist by two-photon polymerization using a Nanoscribe instrument operated in immersion (DILL) mode with $30 \mathrm{~mW}$ power laser and $400 \mathrm{~nm}$ hatching distance and layer height, followed by $15 \mathrm{~min}$ immersion in PGMEA developer and $15 \mathrm{~min}$ post bake at $70^{\circ} \mathrm{C}$.

Two different designs for one and two dimensional CRLs are explored in this paper. First is a new horizontal design in which a 16 blocks of cylindrical lens are placed end to end with a $5 \mu \mathrm{m}$ gap between adjacent lens elements. The $5 \mu \mathrm{m}$ gap served to eliminate the shadowing effect of the laser beam that occurred when writing separate blocks next to each other. Each block is $100 \mu \mathrm{m}$ tall, $100 \mu \mathrm{m}$ wide and $100 \mu \mathrm{m}$ long, and consists of three columns of cylindrical ducts, with each column containing five cylindrical 
ducts stacked horizontally. The cross-section of each duct is $6 \mu \mathrm{m}$ wide and $9 \mu \mathrm{m}$ deep (Figure 2). The x-rays pass through the lenses in parallel with the substrate and will be focused in one direction. This design eliminates the limitation of the height and stitching error Nanoscribe and no absorption will occur due to the substrate thickness (Figure 2).

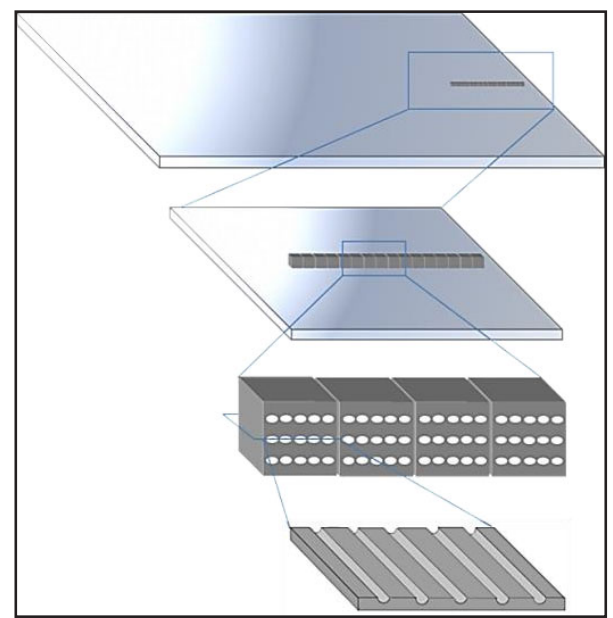

Figure 2. Schematic of the horizontal one dimensional cylindrical $C R L$

The second structure is a linear area of 32 concave lens blocks with $7 \mu \mathrm{m}$ gap (Figure 3). Each block in this design is $100 \mu \mathrm{m}$ tall, $100 \mu \mathrm{m}$ wide, and $50 \mu \mathrm{m}$ long, and contains an array of $3 \times 3$ parabolic indents at both the front and back. Each parabolic surface indent has a depth $\boldsymbol{l}$ of $24 \mu \mathrm{m}$ and a radius $\boldsymbol{r}$ of $6 \mu \mathrm{m}$. This design provides $3 \times 3$ replicate CRL columns, giving $3 \times 3$ replicate images in the microscopy set up to allow measurements of variability among them. The x-rays pass through the lenses in parallel with the substrate and are focused in two dimensions. Based on the refractive index of the photosensitive polymer material, the focal length at $22 \mathrm{keV}$ according to Eq. (4) is $21.5 \mathrm{~mm}$.

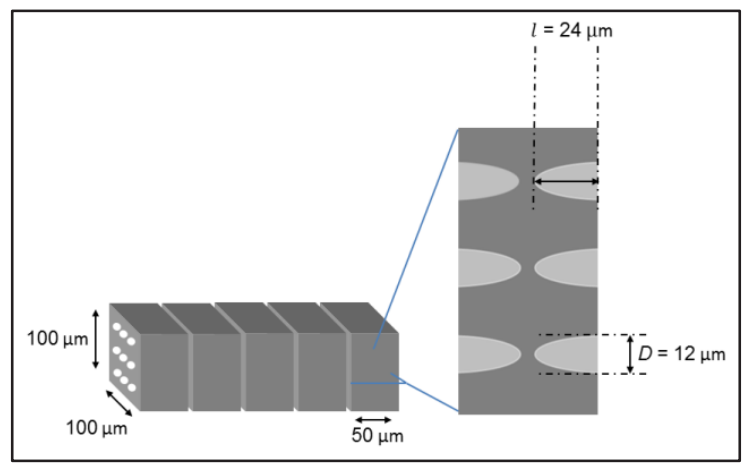

Figure 3. Schematic of the horizontal two dimensional cylindrical $C R L$

Scanning electron microscopy was used to inspect the CRL structures to verify the size of each blocks and the gap between adjacent them. SEM images of one and two dimensional CRLs are shown in Figure 4.
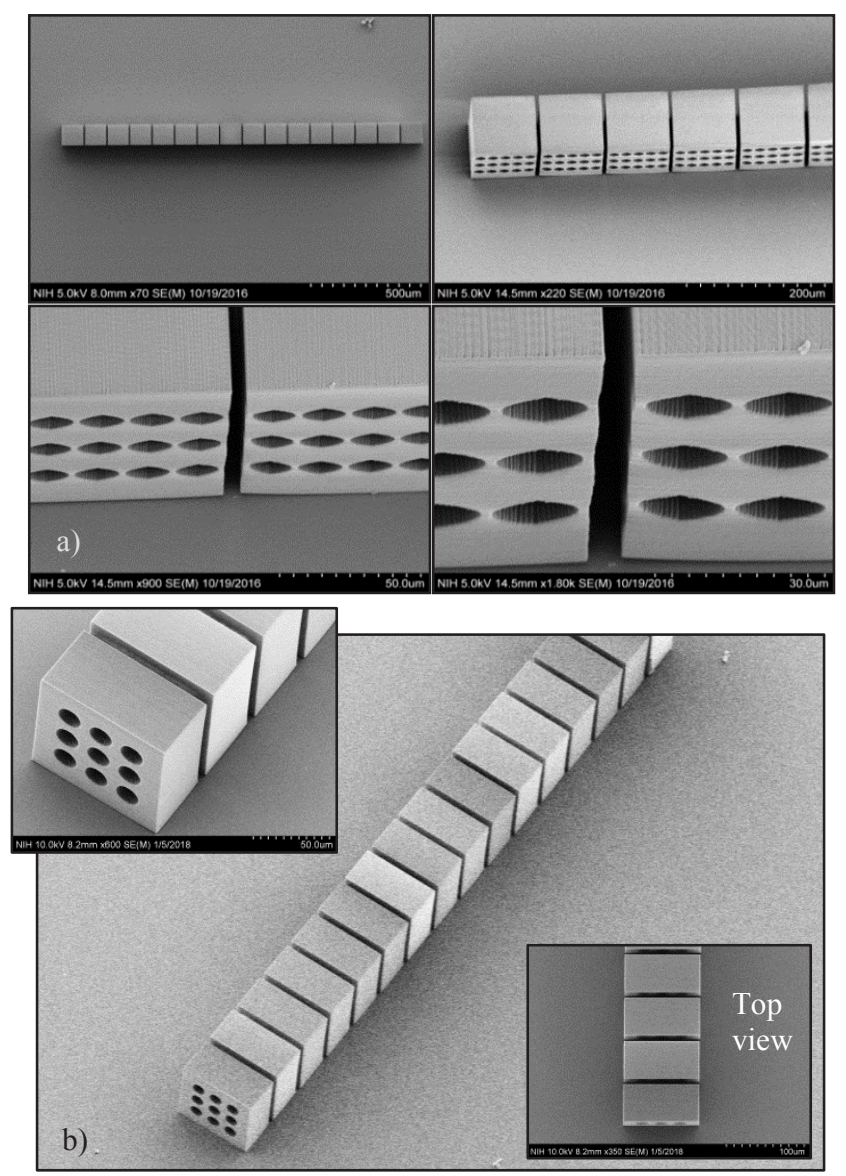

Figure 4. Scanning electron microscopy images of the horizontal (a) one dimensional (b) two dimensional CRL.

\section{Results}

Focusing the x-ray beam of a microCT scanner with cylindrical lenses

A micro-computed tomography (microCT) scanner was used to analyze the energy of the incident radiation and focusing performance of the lens. The Skyscan microCT provides an $11 \mathrm{Mp}$ X-ray camera (12-bit cooled CCD fiber-optically coupled to scintillator) with a micropositioning stage, an array size of $4000 \times 2000$ pixels at the lowest binning, and a $5 \mu \mathrm{m}$ source spot size with $20-100 \mathrm{kV}$ source voltage and $10 \mathrm{~W}$ source power.

Figure 5 shows the focusing effect of the horizontal CRLs in the Skyscan system. The source is operated at $60 \mathrm{keV}$ and $167 \mu \mathrm{A}$ $(10 \mathrm{~W})$. The intensity of the x-ray beam is clearly enhanced while passing through the lenses. The maximum intensity is 250 (A.U.) and lens shows $67 \%$ improvement in x-ray beam focusing effect. 


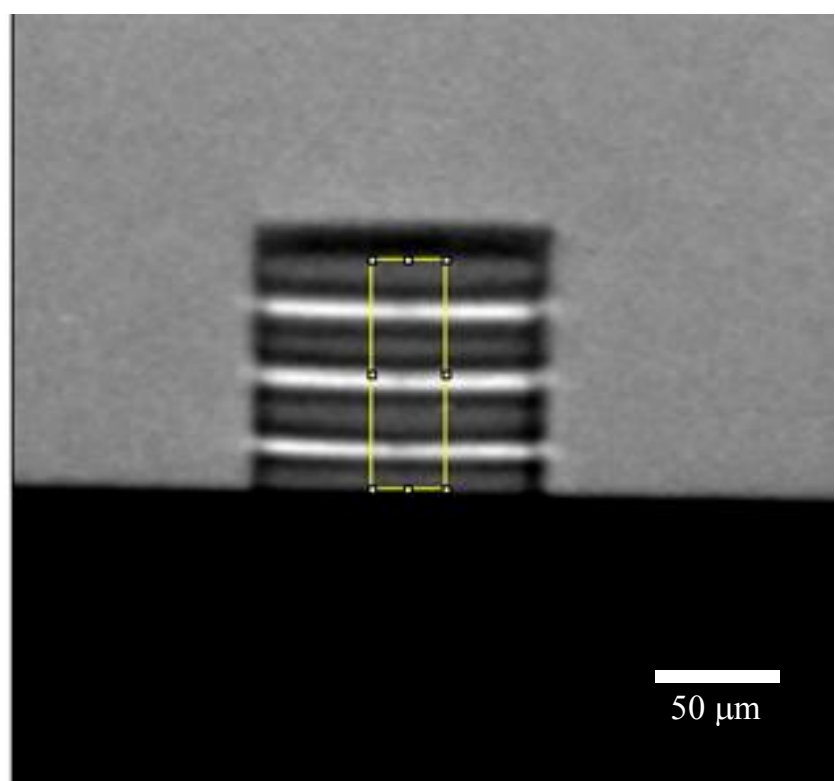

Figure 5. Focusing effect in the cylindrical refractive lens.

\section{Microscopy Test}

We used an experimental x-ray microscopy set up as illustrated in Figure 6, using the nano printed x-ray polymer lens as the magnifying lens. The imaged sample was a $4.8 \mu \mathrm{m}$ period gold grating (Microworks, $\mathrm{GmbH}$, Karlsruhe, Germany). The x-ray source is a fixed-anode, tungsten-target micro focus tube operating at $30 \mathrm{kVp} / 10 \mathrm{~W}$ (Oxford UltraBright 96004, Oxford Instruments, Oxfordshire, United Kingdom), with an average focal spot size of $13.37 \pm 0.35 \mu \mathrm{m}$. An indirect flat panel radiography detector with a pixel size of $83 \mu \mathrm{m}$ (PaxScan 3024M, Varian, CA, USA) was used to image the grating. The geometric distances were $\mathrm{L}_{1}=10 \mathrm{~mm}$ from the source to the sample, $\mathrm{L}_{2}-\mathrm{L}_{1}=20 \mathrm{~mm}$ from the sample to the lens, and $\mathrm{L}_{3}-\mathrm{L}_{2}=1640 \mathrm{~mm}$ from the lens to the detector.

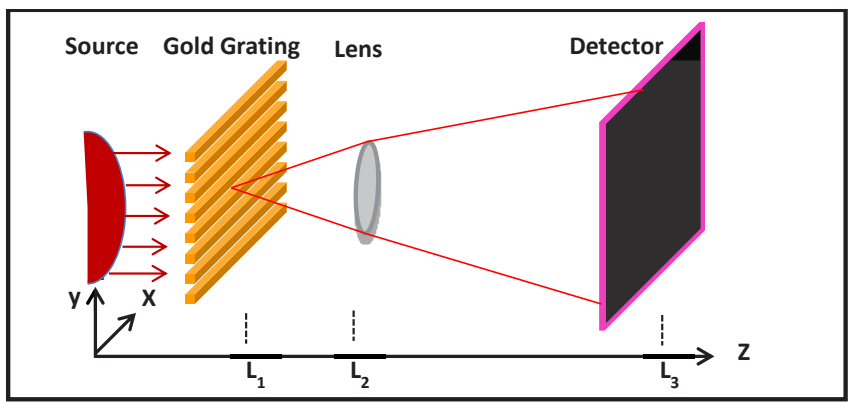

Figure 6. The cone beam from an X-ray tube is exposed to the grating and the diffracted beams are detected at the detector after focusing on the object and passing through the lens.

The gratings were magnified by a factor of $\left(\mathrm{L}_{3}-\mathrm{L}_{2}\right) /\left(\mathrm{L}_{2}-\mathrm{L}_{1}\right)=82$ (Figure 7). The vertical field of view of each CRL approached the $25 \mu \mathrm{m}$ spacing between them. As a result the three groups of grating lines appear to connect with each other. Outside the lens area, direct geometric projection was not able to resolve the grating lines due to the fact that the size of the x-ray tube focal spot is nearly three times the grating period.

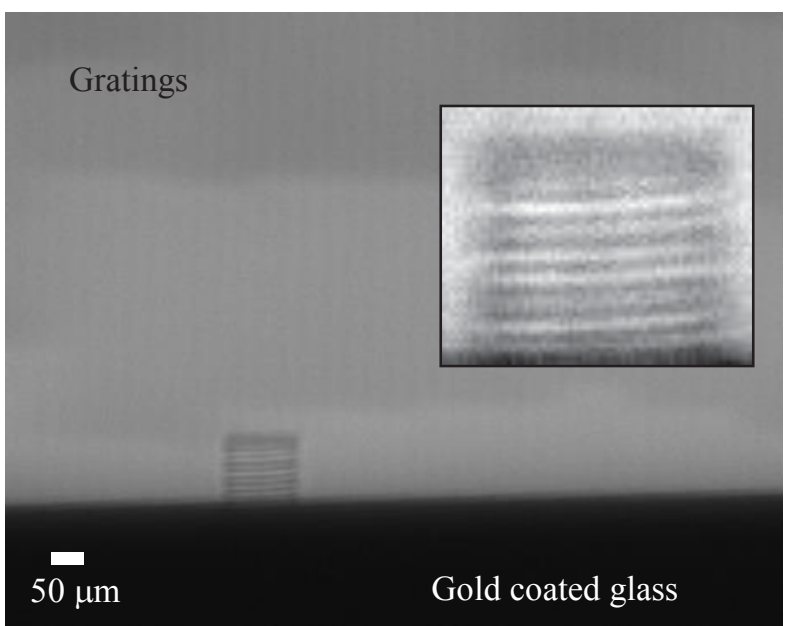

Figure 7. Magnified image of the $4.8 \boldsymbol{\mu} \mathrm{m}$ grating using the micronscale CRL arrays with $19 \mathrm{~mm}$ focal length, $82 x$ magnification.

\section{Imaging and evaluation of $x$-ray focal spots with the concave} CRL

Benchtop imaging of the $\mathrm{x}$-ray focal spot was achieved, and direct study of the lens using the Gaussian fit was provided (Figure 8). The micro-focus X-ray source (Thermo Scientific Kevex) was studied at a range of tube $\mathrm{kV}$ settings and a range of sample-to-lens distances (SLDs) in the imaging setup.
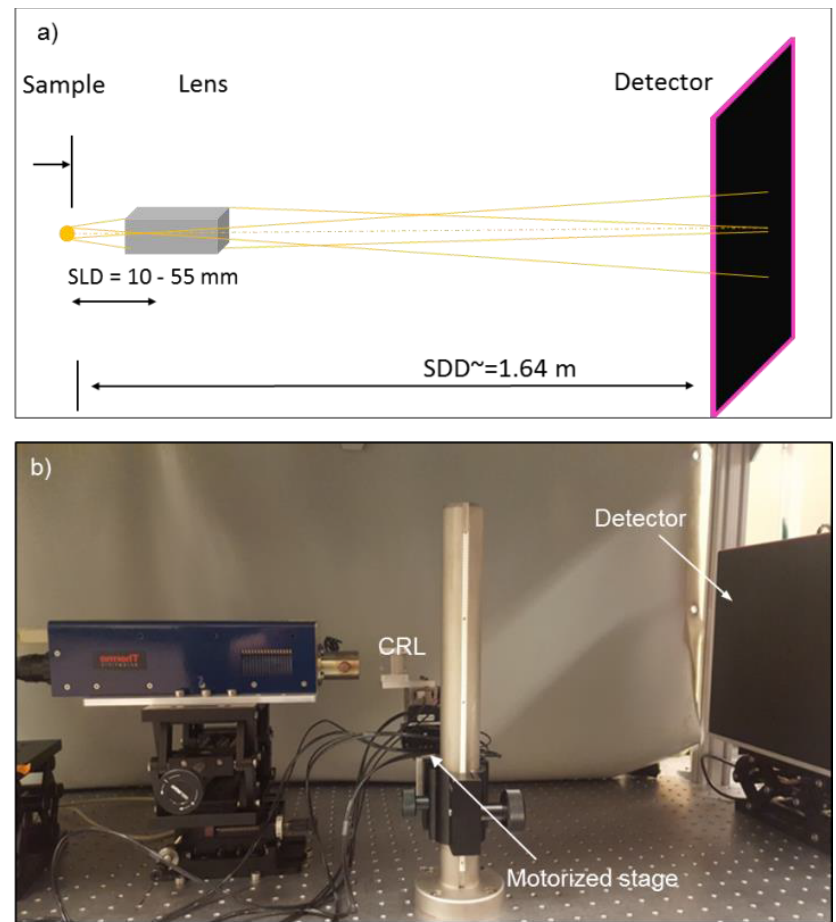

Figure 8. (a) A schematic of the x-ray microscopy setup for imaging the focal spots of $x$-ray sources. (b) In this photograph of an imaging experiment, the focal spot of a tungsten-target microfocus source was being imaged.

The nanofabricated CRL was able to focus the x-ray beam (Figure 9) to exceptionally small length scales as small as $19 \mathrm{~mm}$, 
and maintained focusing at $\mathrm{x}$-ray tube voltages from $25 \mathrm{kV}$ up to $85 \mathrm{kV}$ (Figure 10). Images of the $\mathrm{x}$-ray tube focal spot at $80 \mathrm{x}$ magnification were acquired in seconds. An imaging spot size of 5 $\mu \mathrm{m}$ was measured.

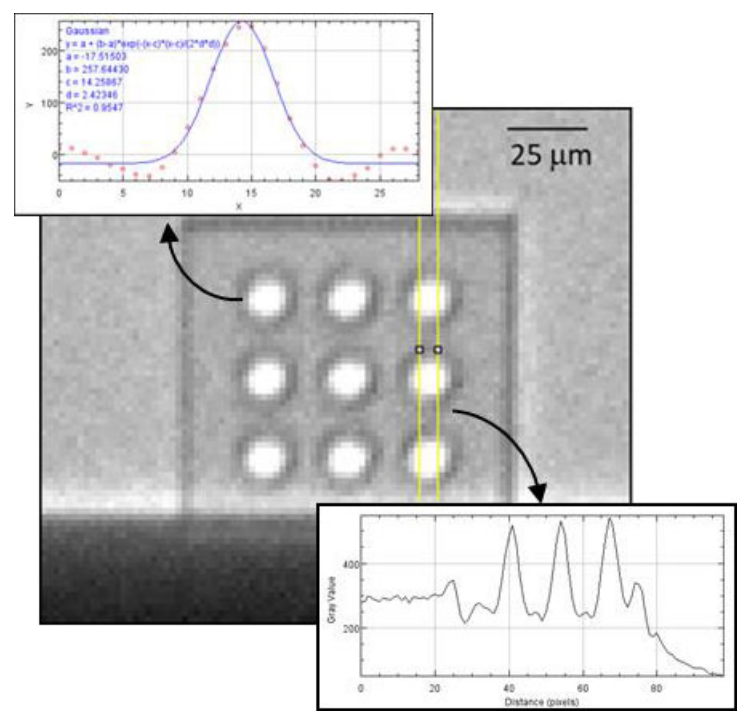

Figure 9. Microscopy image of the $5 \mu \mathrm{m}$ focal spot size of the $x$ ray source.

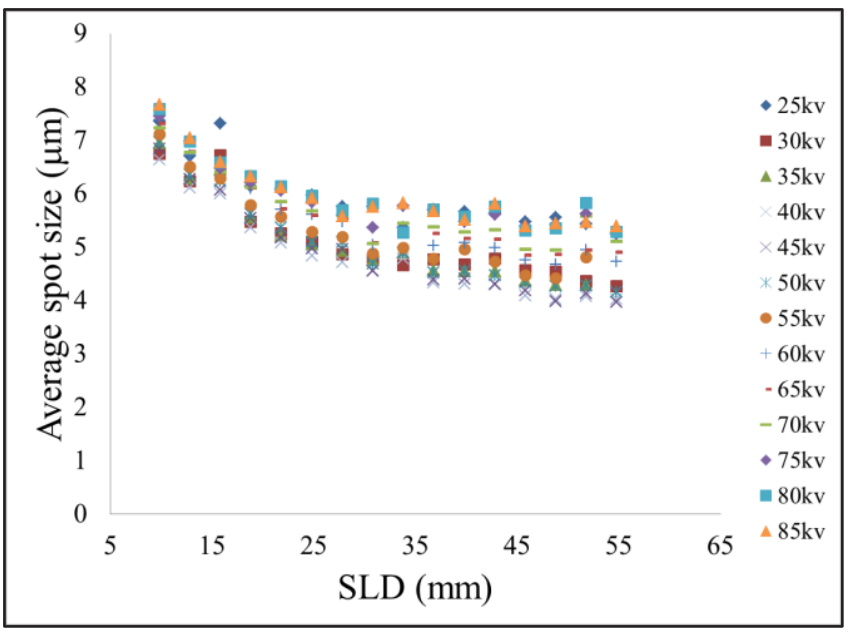

Figure 10. A plot of the average measured focal spot size and standard deviation over the scanned range of SLDs. The standard deviations were $<0.14 \mu \mathrm{m}$ for all measurements.

\section{Discussion}

The results demonstrate that sub-micrometer resolution 3D printing based on two-photon photopolymerization is suitable for producing miniature compound refractive lenses for hard x-rays. The fabricated CRL devices offer the smallest size and shortest focal length that has been reported in the literature for operation with photons in the range of $10 \mathrm{~s}$ of $\mathrm{keVs}$. The devices were able to successfully achieve $19 \mathrm{~mm}$ focal length and $1.1 \mu \mathrm{m}$ pixel resolution. The polymer lens arrays were also shown to be capable of maintaining focus over a range of x-ray tube voltages from 25 $\mathrm{kV}$ to $85 \mathrm{kV}$, and enabling rapid imaging and assessment of the focal spots of tungsten-anode $\mathrm{x}$-ray tubes at up to $159 \mathrm{x}$ magnification.
The measured focal spot size of the x-ray source decreased gradually with increasing SLD, even beyond the designed focal distance (Figure 11). We speculate two possible reasons for the trend. One is that at large SLDs/low magnification, each detector pixel covers a larger area of the focal spot, leading to increasing under-sampling of the spot profile and possible systematic underestimation of the width of the underlying profile. Another possible reason was that the higher parts of the $\mathrm{x}$-ray spectrum $(>22 \mathrm{keV})$ continually come into focus with increasing SLD.

The fabrication time is significantly shorter than the previously reported techniques so that a structure as tall as $1.5 \mathrm{~mm}$ can be written in 2 hours. The nanoprinted lens technology is expected to enable novel benchtop $\mathrm{x}$-ray imaging systems for industrial, material science and medical applications.

\section{References}

[1] Jach T, Durbin SM, Bakulin A, et al. Wide-field x-ray microscopy with Kirkpatrick-Baez optics. In: McNulty I (ed). International Society for Optics and Photonics, pp. $38-44$.

[2] Gary CK, Park H, Lombardo LW, et al. High resolution xray microscope. Appl Phys Lett; 90. Epub ahead of print 2007. DOI: $10.1063 / 1.2734895$.

[3] Snigirev A, Kohn V, Snigireva I, et al. A compound refractive lens for focusing high-energy X-rays. Nature 1996; 384: 49-51.

[4] Dudchik YI, Komarov FF, Piestrup MA, et al. Using of a microcapillary refractive $\mathrm{X}$-ray lens for focusing and imaging. Spectrochim Acta - Part B At Spectrosc 2007; 62: 598-602.

[5] Terentyev S, Polikarpov M, Snigireva I, et al. Linear parabolic single-crystal diamond refractive lenses for synchrotron X-ray sources. In: Journal of Synchrotron Radiation. 2017, pp. 103-109.

[6] Aristov V V., Grigoriev M V., Kuznetsov SM, et al. X-ray focusing by planar parabolic refractive lenses made of silicon. Opt Commun 2000; 177: 33-38.

[7] Etrov AKP, Essonov VOB, Brashitova KAA, et al. Polymer X-ray refractive nano-lenses fabricated by additive technology. 2017; 25: 3256-3259. 